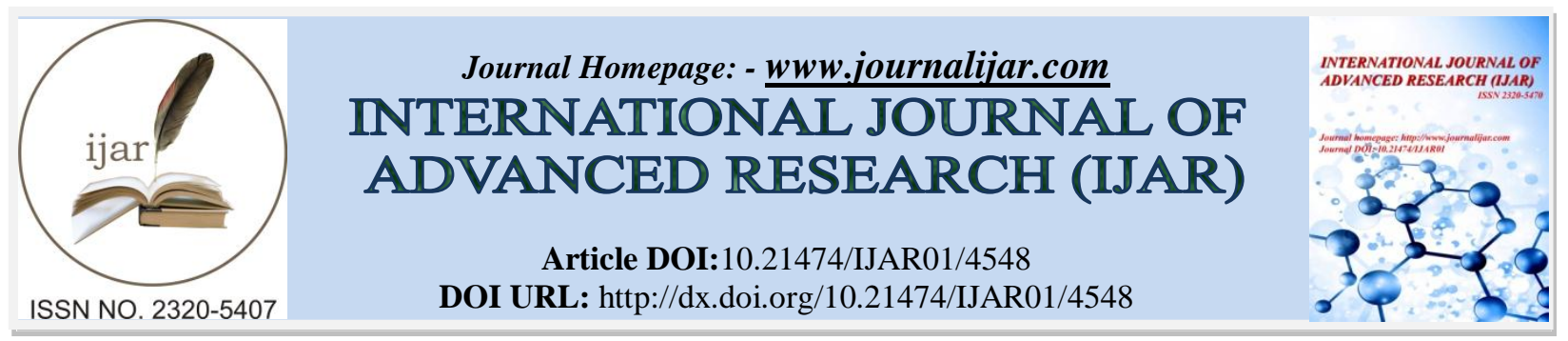

RESEARCH ARTICLE

\title{
STUDY ONMICROBIAL DIVERSITY OF BIOSURFACTANT PRODUCING BACTERIA FROM CONTAMINATED ENVIROMENTAL SAMPLES.
}

\author{
${ }^{*}$ C. C. Okore ${ }^{1}$, L. U. Nwaehiri ${ }^{1}$, O.N. Mbanefo ${ }^{1}$, T. E. Ogbulie ${ }^{2}$, A. U. Ugenyi ${ }^{2}$, I. B. Ogbuka ${ }^{1}$, A. E. Ejele ${ }^{3}$ \\ and I. A. Okwujiako ${ }^{2}$. \\ 1. Environmental Biology Dept, Federal Polytechnic Nekede Owerri, Imo-State. \\ 2. Biotechnology Dept, Federal University of Technology Owerri, Imo-State. \\ 3. Chemistry Dept, Federal University of Technology Owerri, Imo-State.
}

\section{Manuscript Info}

\section{Manuscript History}

Received: 20 April 2017

Final Accepted: 22 May 2017

Published: June 2017

Key words:-

Diversity, biosurfactant, bacteria, environmental samples

\section{Abstract}

Through culture enrichments we isolated nine bacterial genera: Staphylococcus aureus $9.1 \%$, Bacillus sp. 21.2\%, Pseudomonas aeruginosa $27.3 \%$, Corynebacterium spp., 9.1\%, Streptococcus spp., 9.1\%, Micrococcus spp., 12.1\%, Enterobacter spp., 3.0\%, Proteus spp., $6.1 \%$, Escherichia coli $3.0 \%$ from numerous contaminated soils and water samples We characterized the bacterial strains that can utilize different hydrocarbons (diesel, petrol, kerosene, crude oil, vegetable oil) using $\beta$ haemolysis, methylene blue plate assay, drop collapse test, oil displacement and emulsification index; 16 isolates $(66.7 \%)$ produced $\beta$ haemolysis, 6 isolates $(25 \%)$ positive for methylene blue assay, 16 isolates $(66.7 \%)$ collapsed in drop collapsing test. The oil spreading test on diesel, N8 (85 mm, Bacillus sp.) and N12 (80 mm, Pseudomonas sp.) displayed highest values. The emulsification index on diesel N22 (71 mm, Proteus sp), N7 $(67 \mathrm{~mm}$, Pseudomonas sp.) recorded the highest. All strains tested, emulsified the oil to varying degrees and their ability to produce biosurfactant ascertained.

Copy Right, IJAR, 2017,. All rights reserved.

\section{Introduction:-}

Biodiversity generally refers to the variety and variability of life on earth. It is a measure of the variety of organisms present in different ecosystems. This can refer to genetic variation, ecosystem variation, or species variation within an area or biome. Species diversity contributes to ecosystem health. Each species is like a thread holding together an ecosystem. The loss of biodiversity due to human actions has the potential to reduce multi trophic level interactions (Pinto et al., 2014). The variety of consortium of genes that produce biosurfactant can be studied to enhance the degradation of different components of hydrocarbons. The microbial diversity of different species of bacterial producing biosurfactant from environmental contaminated samples is a useful measure of the variety of this same gene that is responsible for biosurfactant production (Bento et al., 2005). Biosurfactant production is a desirable property of hydrocarbon degrading micro organism. In other to obtain efficient hydrocarbon degrading bacteria consortium and monocultures, knowledge of the diversity of the microbial community present in contaminated soil and water samples is important. The isolation of bacteria-producing biosurfactants from the environment can provide excellent materials and resources. The environmental samples are ready raw material for their isolation that is to say that biosurfactant producing bacteria are abundantly available in the environment. 
There are different screening methods for identifying biosurfactant producing bacteria. These include: $\beta$ haemolysis test, methylene blue assay/Centriamide test (CTAB), drop collapsing, oil displacement test and emulsification index test. It is however, difficult to detect the type of biosurfactant produced by the microbes using a single method owing to the chemical and functional properties. In view of this, it appears that several screening methods are needed to understand the ability of a single hydrocarbonoclastic microbe in producing biosurfactant. Hence for efficient detection of potential biosurfactant producers, combinations of various screening methods are required (Satpute et al., 2008). Kiran et al., (2010) also suggested that the single screening method is unsuitable for identifying all types of biosurfactants, and recommended that more than one screening method should be included during primary screening to identify potential biosurfactant producers.

Emulsification of the diesel oil in water is a prerequisite that paves the way for biodegradation of environmental pollutant by many bacteria. It enhances the bioavailability of the oil and thus increases the biodegradation rate (Bredholt et al., 1998; Minf et al., 2011; Hassanshahian et al., 2012). Very often the growth of microorganisms on hydrocarbons is accompanied by the emulsification of the hydrocarbon in the medium, and in most cases this has been attributed to the production of surface-active compounds (Desai and Banat,1997).

A wide variety of metabolic and physiological factors are required for the degradation of different hydrocarbons. All such properties are not found in one organism. Monocultures can be adversely affected by negative interactions. The best approach would be the use of a consortium of biosurfactant producing micro organisms. By selecting a consortium from various contaminated environment, the negative interactions could be minimal (Frielo et al., 2001). The quest for novel bacteria consortium for biosurfactant production lead to this study to identify the diversity of micro organisms from contaminated samples that produce biosurfactants.

\section{Methodology:- \\ Sampling:-}

The crude oil was collected from oil wells, water sample from the fish ponds in Federal Polytechnic Nekede Owerri, water samples from swimming pools, water samples from the Otamiri river, soil samples from palm oil mill, kerosene, petrol and diesel stands, soil from automobile workshop, and diary product (milk). The samples were collected in sterile vials and immediately taken to the Biology Laboratory of Federal Polytechnic Nekede Owerri for analysis.

\section{Cultivation/Inocula Preparation:-}

Samples were serially diluted, dilution of $10^{-2}$ to $10^{-6}$ plated in triplicates by spread plate method on nutrient agar medium and incubated under aerobic conditions at $37^{\circ} \mathrm{C}$ for $24 \mathrm{~h}$. Diesel/crude oil/petrol/kerosene/vegetable oil were each used as hydrocarbon source in each of the Petri-dish with the help of cotton buds and control with no hydrocarbon source was maintained. The medium was enriched with hydrocarbon source in the form of vapours. The viable cell counts of all strains were determined.

\section{Identification Of Isolates:-}

The identification of isolates were carried out using various biochemical tests to find the closest match with known bacterial genus and to assign the bacterial signature according to Bergey's manual of determinative bacteriology. The tests included gram-staining, sugar fermentation test, indole test, citrate test, spore-staining, oxidase test and catalase test.

\section{Screening Tests:- \\ BHaemolysis test:-}

The isolates were streaked on blood agar plates prepared with $5 \mathrm{ml}$ of human blood and $4.2 \mathrm{~g}$ of nutrient agar powder dissolved in $150 \mathrm{ml}$ of water. The blood agar plates were incubated at $30^{\circ} \mathrm{C}$ for $24 \mathrm{~h}$. The occurrence of a define clear zone around the colony is positive indication of biosurfactant production as the biosurfactant lyses the red blood cells (Morikawa, et al., 2000; Youssef et al., 2004).

\section{Methylene blue plate assay:-}

$0.1 \mathrm{ml}$ of methylene blue was introduced into $150 \mathrm{ml}$ of nutrient agar to make methylene blue agar. The plate were incubated at $30^{\circ} \mathrm{C}$ for $48 \mathrm{~h}$. The ability of biosurfactant producers to form clear halos in methylene blue agar plate shows the presence of biosurfactant production (Siegmund and Wagner, 1991; Lin et al., 1998). 


\section{Drop collapsing test}

This is a sensitive rapid method advised for screening bacterial colonies that produce biosurfactants. Drops of supernatant of biosurfactant producing colonies collapsed on oil coated surface (Lin, et al., 1998; Youssef et al., 2004).

\section{Oil spreading:-}

It is a method used to determine the diameter of the clear zone which occurs after adding biosurfactant containing solution on oil-water interphase. The diameter evaluation is correlated to the surface tension reduction efficiency of a given biosurfactant. The distilled water, $25 \mathrm{ml}$ was taken in the large Petri dishes, $0.5 \mathrm{ml}$ of oil was added onto the surface and the centre of the plates containing distilled water. Then $10 \mu \mathrm{l}$ of the supernatant of the cultures isolated from the samples was added to the centre of the oil. The biosurfactant producing organisms displaced the oil and spread in the water. The diameter and the clear halo visualized under visible light was measured after 30 sec and results taken (Fiebigi, et al., 1997; Maneerat, et al., 2005).

\section{Emulsification test:-}

Emulsification capacity of the biosurfactant towards hydrocarbons (diesel, petrol, vegetable oil, kerosene, crude oil) was carried out using a mixture of $2 \mathrm{ml}$ oil added to the same amount of cell free supernatant obtained after vortexing sample culture grown on nutrient broth, at high speed for $2 \mathrm{~min}$ and left to stand for $24 \mathrm{~h}$. The emulsification index $\left(\mathrm{E}_{24}\right)$ was calculated as the percentage of height of the emulsified layer (mm) divided by total height of the liquid/aqueous column (mm) (Yuste et al.,2000; Schulz et al., 1991).

\section{Result:-}

Table 1:- Total viable counts for all samples

\begin{tabular}{|c|c|c|c|c|}
\hline $\mathbf{S} / \mathbf{N}$ & & Viable count & & Total viable count (cfu) \\
\hline 1 & Soil from palm oil mill & $\begin{array}{lr}\text { PC1 } & \text { PC2 } \\
3.9 \times 10^{5} & 2.8 \times 10^{5}\end{array}$ & $\begin{array}{r}\mathrm{PC} 3 \\
1.2 \times 10^{4}\end{array}$ & $6.8 \times 10^{5}$ \\
\hline 2 & Crude oil & $\begin{array}{lc}\text { C1 } & \text { C2 } \\
2.7 \times 10^{4} & 0.7 \times 10^{4} \\
\end{array}$ & $\begin{array}{c}\text { C3 } \\
1.1 \times 10^{4}\end{array}$ & $4.5 \times 10^{4}$ \\
\hline 3 & Water from Otmiri river & $\begin{array}{ll}\mathrm{R} 1 & \mathrm{R} 2 \\
1.0 \times 10^{4} & 6.0 \times 10^{4} \\
\end{array}$ & $\begin{array}{c}\text { R3 } \\
4.5 \times 10^{4}\end{array}$ & $11.5 \times 10^{4}$ \\
\hline 4 & Soil near kerosene Pump & $\begin{array}{ll}\text { K1 } & \text { K2 } \\
1.9 \times 10^{4} & 2.1 \times 10^{4} \\
\end{array}$ & $\begin{array}{c}\mathrm{K} 3 \\
5.0 \times 10^{4}\end{array}$ & $9.0 \times 10^{4}$ \\
\hline 5 & Soil near diesel Pump & $\begin{array}{lc}\text { D1 } & \text { D2 } \\
1.7 \times 10^{4} & 1.8 \times 10^{4} \\
\end{array}$ & $\begin{array}{c}\mathrm{K} 3 \\
1.9 \times 10^{4} \\
\end{array}$ & $5.4 \times 10^{4}$ \\
\hline 6 & Soil near petrol pump & $\begin{array}{lc}\text { F1 } & \text { F2 } \\
1.7 \times 10^{4} & 9.0 \times 10^{4} \\
\end{array}$ & $\begin{array}{c}\text { F3 } \\
1.8 \times 10^{4} \\
\end{array}$ & $12.5 \times 10^{4}$ \\
\hline 7 & $\begin{array}{l}\text { Water from swimming } \\
\text { pool }\end{array}$ & $\begin{array}{lc}\text { S1 } & \text { S2 } \\
2.9 \times 10^{5} & 2.5 \times 10^{5}\end{array}$ & $\begin{array}{l}\mathrm{S} 3 \\
2.4 \times 10^{5}\end{array}$ & $7.8 \times 10^{5}$ \\
\hline 8 & $\begin{array}{l}\text { Automobile workshop } \\
\text { soil }\end{array}$ & $\begin{array}{lr}\text { AA1 } & \text { AA2 } \\
5.1 \times 10^{4} & 1.5 \times 10^{4} \\
\end{array}$ & $\begin{array}{c}\mathrm{AA} 3 \\
1.1 \times 10^{4} \\
\end{array}$ & $8.7 \times 10^{4}$ \\
\hline 9 & Water from fish pond & $\begin{array}{ll}\text { FP1 } & \text { FP2 } \\
1.4 \times 10^{4} & 0.8 \times 10^{4} \\
\end{array}$ & $\begin{array}{c}\text { FP3 } \\
3.0 \times 10^{4}\end{array}$ & $5.2 \times 10^{4}$ \\
\hline 10 & Milk & $\begin{array}{cc}\text { M1 } & \mathrm{M} 2 \\
2.0 \times 10^{4} & 1.0 \times 10^{4} \\
\end{array}$ & $\begin{array}{r}\mathrm{M3} \\
4.7 \times 10^{4}\end{array}$ & $7.7 \times 10^{4}$ \\
\hline 11 & Abattoir & $\begin{array}{cc}\mathrm{AB} 1 & \mathrm{AB} 2 \\
3.0 \times 10^{4} & 2.0 \times 10^{4}\end{array}$ & $\begin{array}{r}\mathrm{AB} 3 \\
4.7 \times 10^{4}\end{array}$ & $9.7 \times 10^{4}$ \\
\hline 12 & Soil from paint industry & $\begin{array}{lr}\text { PA1 } & \text { PA2 } \\
2.7 \times 10^{4} & 1.1 \times 10^{4} \\
\end{array}$ & $\begin{array}{l}\text { PA3 } \\
2.8 \times 10^{4}\end{array}$ & $6.6 \times 10^{4}$ \\
\hline
\end{tabular}

Key: $\mathrm{PC} 1, \mathrm{PC} 2, \mathrm{PC} 3=$ Triplicates of soil from palm oil mill

C1, C2, C3 = Triplicates of crude oil sample

R1, R2, R3 = Triplicates of Otamiri river water sample

$\mathrm{K} 1, \mathrm{~K} 2, \mathrm{~K} 3$ = Triplicates of soil near kerosene pump

D1, D2, D3 = Triplicates of soil near diesel pump

F1, F2, F3 = Triplicates of soil near petrol pump

S1, S2, S3 = Triplicates of swimming pool water sample 
AA1, AA2, AA3 =Triplicates of automobile soil sample

FP1, FP2, FP3, = Triplicates of fish pond water sample

M1, M2, M3 = Triplicates of milk sample

$\mathrm{AB} 1, \mathrm{AB} 2, \mathrm{AB} 3=$ Triplicates of soil from abattoir

PA1, PA2, PA3 = Triplicates of soil sample from paint industry

Table 2:- Biochemical test for identification of isolates.

\begin{tabular}{|c|c|c|c|c|c|c|c|c|c|c|c|c|c|c|}
\hline \multirow[b]{2}{*}{ 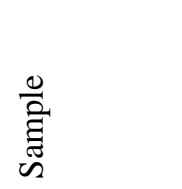 } & \multirow[b]{2}{*}{ 营 } & \multirow[b]{2}{*}{$\sum_{i=\frac{\pi}{0}}^{0}$} & \multirow{2}{*}{$\begin{array}{l}\text { Morphological } \\
\text { Characteristics }\end{array}$} & \multirow{2}{*}{ 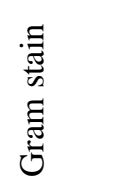 } & \multirow{2}{*}{ 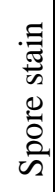 } & \multirow[b]{2}{*}{ 苛 } & \multirow[b]{2}{*}{$\begin{array}{l}\frac{0}{0} \\
\frac{0}{g}\end{array}$} & \multirow[b]{2}{*}{ 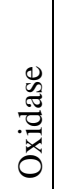 } & \multirow{2}{*}{ 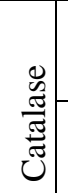 } & \multicolumn{4}{|c|}{ Sugar test } & \multirow{2}{*}{$\begin{array}{l}\text { Possible } \\
\text { Bacteria }\end{array}$} \\
\hline & & & & & & & & & & $\mathbf{S}$ & B & $\mathbf{G}$ & $\mathrm{H}_{2} \mathrm{~S}$ & \\
\hline $\begin{array}{l}\text { PA1, PA2, } \\
\text { PA3 }\end{array}$ & 1 & NA & $\begin{array}{l}\text { Milkish raised } \\
\text { non-mucoid. } \\
\text { Circular } \\
\text { colonies }\end{array}$ & $\begin{array}{l}+\mathrm{ve} \\
\text { cocci }\end{array}$ & - & - & - & - & + & $\mathrm{R}$ & $\mathbf{Y}$ & - & - & $\begin{array}{l}\text { Staphylococcus } \\
\text { aureus }\end{array}$ \\
\hline PA1 & 2 & NA & $\begin{array}{l}\text { Milkish flat } \\
\text { non-mucoid } \\
\text { colonies with } \\
\text { undulate edges. }\end{array}$ & $\begin{array}{l}+\mathrm{ve} \\
\text { rod }\end{array}$ & - & - & - & - & + & $\mathrm{R}$ & $\mathbf{Y}$ & - & - & Bacillus spp. \\
\hline PA1, PA2 & 3 & NA & $\begin{array}{l}\text { Bluish-green } \\
\text { pigmented non- } \\
\text { mucoid } \\
\text { colonies }\end{array}$ & $\begin{array}{l}- \text {-ve } \\
\text { Rod }\end{array}$ & - & - & - & + & - & $\mathrm{Y}$ & $\mathbf{Y}$ & - & - & $\begin{array}{l}\text { Pseudomonas } \\
\text { aeruginosa }\end{array}$ \\
\hline $\begin{array}{l}\text { PA1, PA2, } \\
\text { PC1, AC3, } \\
\text { PB2, AC2 }\end{array}$ & 4 & NA & $\begin{array}{l}\text { Milkish raised } \\
\text { needle pointed } \\
\text { non-mucoid } \\
\text { colonies }\end{array}$ & $\begin{array}{l}+\mathrm{ve} \\
\text { cocci } \\
\text { in } \\
\text { chain }\end{array}$ & - & - & + & - & - & $\mathrm{Y}$ & $\mathbf{Y}$ & - & - & $\begin{array}{l}\text { Corynebacterium } \\
\text { spp. }\end{array}$ \\
\hline $\begin{array}{l}\text { PA2, AC2, } \\
\text { PB3, }\end{array}$ & 5 & NA & $\begin{array}{l}\text { Milkish flat } \\
\text { non-mucoid } \\
\text { colonies with } \\
\text { rough edge }\end{array}$ & $\begin{array}{l}+\mathrm{ve} \\
\text { cocci }\end{array}$ & - & - & - & + & - & $\mathrm{R}$ & $\mathbf{Y}$ & - & - & Bacillus spp. \\
\hline $\begin{array}{l}\text { PA3,PB1, } \\
\text { PB2 } \\
\text { PB3,PC2,P } \\
\text { C1, } \\
\text { AB1,AB2, } \\
\text { AB3 }\end{array}$ & 6 & NA & $\begin{array}{l}\text { Bluish-green } \\
\text { pigmented } \\
\text { rhizoid-like } \\
\text { colonies }\end{array}$ & $\begin{array}{l}+\mathrm{ve} \\
\text { cocci }\end{array}$ & - & - & - & + & - & $\mathrm{R}$ & $\mathbf{Y}$ & - & - & $\begin{array}{l}\text { Pseudomonas } \\
\text { aeruginosa }\end{array}$ \\
\hline $\begin{array}{l}\text { PA3,PB1, } \\
\text { PC3, }\end{array}$ & 7 & NA & $\begin{array}{l}\text { Milkish flat } \\
\text { non-mucoid } \\
\text { colonies with } \\
\text { undulate edges. }\end{array}$ & $\begin{array}{l}+\mathrm{ve} \\
\text { cocci }\end{array}$ & - & - & - & + & $=$ & $\mathrm{R}$ & $\mathbf{Y}$ & - & - & Bacillus spp. \\
\hline PB2 & 8 & NA & $\begin{array}{l}\text { Milkish flat } \\
\text { rhizoid-like } \\
\text { colonies }\end{array}$ & $\begin{array}{l}+\mathrm{ve} \\
\text { rod }\end{array}$ & + & - & - & - & - & $\mathrm{R}$ & $\mathbf{Y}$ & - & - & $\begin{array}{l}\text { Pseudomonas } \\
\text { aeruginosa }\end{array}$ \\
\hline PB2 & 9 & NA & $\begin{array}{l}\text { Milkish flat } \\
\text { non-mucoid } \\
\begin{array}{l}\text { colonies with } \\
\text { zone } \\
\text { clearance }\end{array}\end{array}$ & $\begin{array}{l}+\mathrm{ve} \\
\text { rod }\end{array}$ & - & - & - & - & - & $\mathrm{R}$ & $\mathbf{Y}$ & - & - & $\begin{array}{l}\text { Streptoccoccus } \\
\text { spp }\end{array}$ \\
\hline
\end{tabular}


Table 3:- Biochemical test for identification of isolates.

\begin{tabular}{|c|c|c|c|c|c|c|c|c|c|c|c|c|c|c|}
\hline \multirow{2}{*}{ 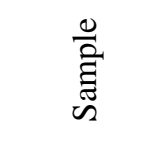 } & \multirow{2}{*}{\begin{tabular}{l}
$\stackrel{\mathscr{\pi}}{\pi}$ \\
\hdashline \\
$\mathscr{E}$
\end{tabular}} & \multirow{2}{*}{ 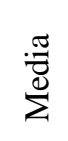 } & \multirow[b]{2}{*}{$\begin{array}{l}\text { Morphological } \\
\text { Chatacteristics }\end{array}$} & \multirow{2}{*}{ 芯 } & \multirow{2}{*}{ 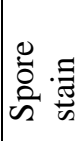 } & \multirow{2}{*}{ 芯 } & \multirow{2}{*}{ 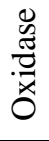 } & \multirow{2}{*}{$\frac{0}{0}$} & \multirow{2}{*}{$\frac{\mathscr{E}}{\frac{\tilde{J}}{\pi}}$} & \multicolumn{4}{|c|}{ Sugar test } & \multirow[b]{2}{*}{$\begin{array}{l}\text { Possible } \\
\text { bacteria }\end{array}$} \\
\hline & & & & & & & & & & $\mathbf{S}$ & B & $\mathbf{G}$ & $\mathbf{H}_{2} \mathrm{~S}$ & \\
\hline $\mathrm{AA} 3, \mathrm{P} 2$ & 10 & N.A & $\begin{array}{l}\text { Milkish } \\
\text { enlarged non } \\
\text { mucoid } \\
\text { colonies }\end{array}$ & $\begin{array}{l}+\mathrm{ve} \\
\text { cocci }\end{array}$ & - & - & - & - & + & $\mathrm{R}$ & $\mathbf{Y}$ & - & - & $\begin{array}{l}\text { Micrococcus } \\
\text { spp. }\end{array}$ \\
\hline AA3, PB2 & 11 & N.A & $\begin{array}{l}\text { Milkish raised } \\
\text { non-mucoid } \\
\text { colony }\end{array}$ & $\begin{array}{l}+\mathrm{ve} \\
\text { cocci }\end{array}$ & - & + & - & - & - & $\mathrm{R}$ & $\mathbf{Y}$ & - & - & Bacillus spp. \\
\hline $\begin{array}{l}\text { AB1, } \\
\text { AB3 }\end{array}$ & 12 & N.A & $\begin{array}{l}\text { Bluish-green } \\
\text { pigmented } \\
\text { colonies with } \\
\text { zone of } \\
\text { clearance }\end{array}$ & $\begin{array}{l}\text { +ve } \\
\text { cocci }\end{array}$ & - & + & - & + & + & $\mathrm{Y}$ & $\mathbf{Y}$ & + & - & $\begin{array}{l}\text { Pseudomonas } \\
\text { spp. }\end{array}$ \\
\hline $\mathrm{PC} 2$ & 13 & N.A & $\begin{array}{l}\text { Golden yellow } \\
\text { flat circular } \\
\text { non-mucoid } \\
\text { colonies }\end{array}$ & $\begin{array}{l}\text { +ve } \\
\text { rod }\end{array}$ & + & - & - & - & - & $\mathrm{R}$ & $\mathbf{Y}$ & + & - & $\begin{array}{l}\text { Streptococcus } \\
\text { spp. }\end{array}$ \\
\hline $\mathrm{AC} 3$ & 14 & N.A & $\begin{array}{l}\text { Milkish } \\
\text { enlarged flat } \\
\text { non-mucoid } \\
\text { colonies with } \\
\text { irregular edges }\end{array}$ & $\begin{array}{l}\text { +ve } \\
\text { rod }\end{array}$ & + & - & - & - & + & $\mathrm{R}$ & $\mathbf{Y}$ & - & - & $\begin{array}{l}\text { Micrococcus } \\
\text { spp. }\end{array}$ \\
\hline $\mathrm{K} 1, \mathrm{~K} 2$ & 15 & N.A & $\begin{array}{l}\text { Milkish flat } \\
\text { non-mucoid } \\
\text { colonies with } \\
\text { rough edges }\end{array}$ & $\begin{array}{l}\text {-ve } \\
\text { rod }\end{array}$ & - & + & - & - & + & $\mathrm{Y}$ & $\mathbf{Y}$ & +2 & - & $\begin{array}{l}\text { Enterobacteria } \\
\text { spp. }\end{array}$ \\
\hline $\begin{array}{l}\text { R1,R2,R3, } \\
\text { M1,M3, } \\
\text { D3, } \\
\text { F3,C3 }\end{array}$ & 16 & N.A & $\begin{array}{l}\begin{array}{l}\text { Milkish flat } \\
\text { enlarged non- } \\
\text { mucoid }\end{array} \\
\text { colonies with } \\
\text { irregular edges }\end{array}$ & $\begin{array}{l}\text { +ve } \\
\text { rod }\end{array}$ & - & + & - & - & - & $\mathrm{R}$ & $\mathbf{Y}$ & - & - & $\begin{array}{l}\text { Micrococcus } \\
\text { spp. }\end{array}$ \\
\hline $\mathrm{S} 1, \mathrm{~S} 2, \mathrm{~S} 3$ & 17 & N.A & $\begin{array}{l}\text { Milkish } \\
\text { elongated non- } \\
\text { mucoid } \\
\text { colonies } \\
\end{array}$ & $\begin{array}{l}\text { +ve } \\
\text { rod }\end{array}$ & - & + & + & - & + & $\mathrm{Y}$ & $\mathbf{Y}$ & $+^{2}$ & - & Proteus spp. \\
\hline D1 & 18 & N.A & $\begin{array}{l}\text { Bluish-green } \\
\text { flat mucoid } \\
\text { colonies with } \\
\text { serrated edges }\end{array}$ & $\begin{array}{l}\text {-ve } \\
\text { cocci }\end{array}$ & - & + & + & - & - & $\mathrm{Y}$ & $\mathbf{Y}$ & + & - & $\begin{array}{l}\text { Pseudomonas } \\
\text { spp. }\end{array}$ \\
\hline D2 & 19 & N.A & $\begin{array}{l}\text { Milkish flat } \\
\text { enlarged non- } \\
\text { mucoid } \\
\text { colonies with } \\
\text { hard-rough } \\
\text { edges }\end{array}$ & $\begin{array}{l}\text {-ve } \\
\text { rod }\end{array}$ & - & + & - & - & - & $\mathrm{R}$ & $\mathbf{R}$ & + & - & Proteus spp. \\
\hline
\end{tabular}


Table 4:- Biochemical test for identification of isolates.

\begin{tabular}{|c|c|c|c|c|c|c|c|c|c|c|c|c|c|c|}
\hline \multirow[b]{2}{*}{ 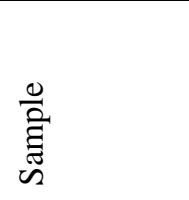 } & \multirow[b]{2}{*}{$\begin{array}{l}\stackrel{\mathscr{J}}{\pi} \\
\stackrel{0}{=}\end{array}$} & \multirow[b]{2}{*}{$\sum_{\substack{0 \\
巳}}^{\frac{\pi}{2}}$} & \multirow{2}{*}{$\begin{array}{l}\text { Morphological } \\
\text { Characteristics }\end{array}$} & \multirow{2}{*}{ 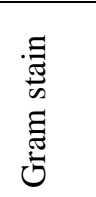 } & \multirow{2}{*}{ 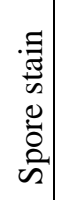 } & \multirow[b]{2}{*}{ : } & \multirow[b]{2}{*}{$\frac{\frac{0}{0}}{\frac{\sigma}{\Xi}}$} & \multirow{2}{*}{\multicolumn{2}{|c|}{ 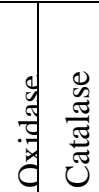 }} & \multicolumn{4}{|c|}{ Sugar test } & \multirow{2}{*}{$\begin{array}{l}\text { Possible } \\
\text { Bacteria }\end{array}$} \\
\hline & & & & & & & & & & $\mathbf{S}$ & $\mathbf{B}$ & $\mathbf{G}$ & $\mathrm{H}_{2} \mathrm{~S}$ & \\
\hline $\mathrm{C} 2$ & 20 & NA & $\begin{array}{l}\text { Bluish-green } \\
\text { flat non-mucoid } \\
\text { colonies }\end{array}$ & $\begin{array}{l}+\mathrm{ve} \\
\text { cocci }\end{array}$ & - & - & - & - & + & $\mathrm{Y}$ & $\mathbf{Y}$ & + & - & $\begin{array}{l}\text { Pseudomonas } \\
\text { spp. }\end{array}$ \\
\hline $\mathrm{R} 1, \mathrm{R} 2$ & 21 & NA & $\begin{array}{l}\text { Bluish-green } \\
\text { flat non-mucoid } \\
\text { colonies with } \\
\text { rough edges. }\end{array}$ & $\begin{array}{l}\text { +ve } \\
\text { cocci }\end{array}$ & - & - & - & - & + & $\mathrm{R}$ & $\mathbf{Y}$ & - & - & $\begin{array}{l}\text { Pseudomonas } \\
\text { spp. }\end{array}$ \\
\hline $\begin{array}{l}\text { R1, FP1, } \\
\text { M1, M3, } \\
\text { K1, K2, } \\
\text { F3 }\end{array}$ & 22 & NA & $\begin{array}{l}\text { Milkish flat. } \\
\text { mucoid colonies } \\
\text { with smooth } \\
\text { circular edges }\end{array}$ & $\begin{array}{l}\text { +ve } \\
\text { cocci }\end{array}$ & + & - & - & - & - & $\mathrm{Y}$ & $\mathbf{Y}$ & - & - & $\begin{array}{l}\text { Escherichia } \\
\text { Coli }\end{array}$ \\
\hline $\begin{array}{l}\text { R3, FP3, } \\
\text { FP1 }\end{array}$ & 23 & NA & $\begin{array}{l}\text { Milkish flat } \\
\text { cottony non- } \\
\text { mucoid colonies }\end{array}$ & $\begin{array}{l}\text { +ve } \\
\text { rod }\end{array}$ & + & - & - & - & + & $\mathrm{R}$ & $\mathbf{Y}$ & - & - & $\begin{array}{l}\text { Bacillus } \\
\text { spp. }\end{array}$ \\
\hline R3, F3, & 24 & NA & $\begin{array}{l}\text { Milkish flat non- } \\
\text { mucoid colonies } \\
\text { with serrated edges }\end{array}$ & $\begin{array}{l}\text { +ve } \\
\text { rod }\end{array}$ & + & - & - & - & - & $\mathrm{R}$ & $\mathbf{Y}$ & - & - & $\begin{array}{l}\text { Bacillus } \\
\text { spp. }\end{array}$ \\
\hline R3, FP3, & 25 & NA & $\begin{array}{l}\text { Milkish raised } \\
\text { needle pointed } \\
\text { non- mucoid } \\
\text { colonies }\end{array}$ & $\begin{array}{l}\text { +ve } \\
\text { rod }\end{array}$ & - & - & - & - & + & $\mathrm{R}$ & $\mathbf{Y}$ & - & - & $\begin{array}{l}\text { Corynebacterium } \\
\text { spp. }\end{array}$ \\
\hline FP2 & 26 & NA & $\begin{array}{l}\text { Milkish raised } \\
\text { needle-like non- } \\
\text { mucoid colonies }\end{array}$ & $\begin{array}{l}\text { +ve } \\
\text { rod }\end{array}$ & - & - & - & - & - & $\mathrm{R}$ & $\mathbf{Y}$ & - & - & $\begin{array}{l}\text { Corynebacterium } \\
\text { spp. }\end{array}$ \\
\hline $\begin{array}{l}\text { C1,C3,K1, } \\
\text { F1,FP1, } \\
\text { M3, } \\
\text { M2. }\end{array}$ & 27 & NA & $\begin{array}{l}\text { Golden yellow } \\
\text { raised mucoid } \\
\text { colonies }\end{array}$ & $\begin{array}{l}\text { +ve } \\
\text { cocci }\end{array}$ & - & + & - & - & + & $\mathrm{Y}$ & $\mathbf{Y}$ & + & - & $\begin{array}{l}\text { Staphylococcus } \\
\text { aureus }\end{array}$ \\
\hline $\begin{array}{l}\text { C1,C3,R1, } \\
\text { R2,R3,M1, } \\
\text { M2,D3,F3 }\end{array}$ & 28 & NA & $\begin{array}{l}\text { Milkish enlarged } \\
\text { non-mucoid } \\
\text { colonies }\end{array}$ & $\begin{array}{l}\text {-ve } \\
\text { rod }\end{array}$ & - & + & - & + & - & $\mathrm{R}$ & $\mathbf{Y}$ & - & - & Micrococcus spp. \\
\hline $\begin{array}{l}\text { C1, FP1, } \\
\text { FP3 }\end{array}$ & 29 & NA & $\begin{array}{l}\text { Bluish-green flat } \\
\text { emerged non- } \\
\text { mucoid colonies }\end{array}$ & $\begin{array}{l}\text { +ve } \\
\text { cocci }\end{array}$ & - & + & - & + & + & $\mathrm{Y}$ & $\mathbf{Y}$ & - & - & $\begin{array}{l}\text { Pseudomonas } \\
\text { aeruginosa. }\end{array}$ \\
\hline C3, R3 & 30 & NA & $\begin{array}{l}\text { Bluish-green } \\
\text { pigmented rhizoid- } \\
\text { like colonies. }\end{array}$ & $\begin{array}{l}\text { +ve } \\
\text { rod }\end{array}$ & - & + & + & + & + & $\mathrm{R}$ & $\mathbf{Y}$ & - & - & $\begin{array}{l}\text { Pseudomonas } \\
\text { aeruginosa }\end{array}$ \\
\hline $\begin{array}{l}\text { C1,R2,K2, } \\
\text { S3,S1,FP1, } \\
\text { M1 }\end{array}$ & 31 & NA & $\begin{array}{l}\text { Milkish-raised non- } \\
\text { mucoid circular } \\
\text { colonies }\end{array}$ & $\begin{array}{l}\text { +ve } \\
\text { rod }\end{array}$ & - & + & - & - & + & $\mathrm{R}$ & $\mathbf{Y}$ & - & - & $\begin{array}{l}\text { Staphylococcus } \\
\text { aureus }\end{array}$ \\
\hline $\begin{array}{l}\text { C2, R2, R3, } \\
\text { M2, }\end{array}$ & 32 & NA & $\begin{array}{l}\text { Milkish flat non- } \\
\text { mucoid colonies } \\
\text { with zone of } \\
\text { clearance }\end{array}$ & $\begin{array}{l}\text { +ve } \\
\text { rod }\end{array}$ & - & - & - & - & + & $\mathrm{R}$ & $\mathbf{Y}$ & - & - & $\begin{array}{l}\text { Streptococcus } \\
\text { spp. }\end{array}$ \\
\hline $\begin{array}{l}\mathrm{C} 3, \mathrm{M} 1, \\
\mathrm{~K} 1, \mathrm{~K} 2\end{array}$ & 33 & NA & $\begin{array}{l}\text { Milkish flat non- } \\
\text { mucoid colonies } \\
\text { with rough edges. }\end{array}$ & $\begin{array}{l}\text { +ve } \\
\text { rod }\end{array}$ & + & - & - & - & + & $\mathrm{R}$ & $\mathbf{Y}$ & - & - & Bacillus spp. \\
\hline
\end{tabular}


Table 5:- Screening tests of bacteria isolates for biosurfactant production.

\begin{tabular}{|c|c|c|c|c|c|c|c|c|c|c|c|c|c|c|}
\hline \multirow[b]{2}{*}{ 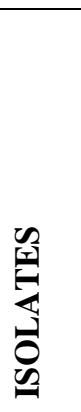 } & \multirow[b]{2}{*}{ 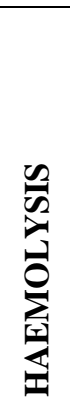 } & \multirow{2}{*}{ 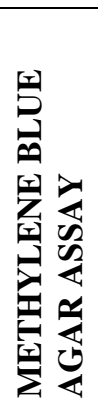 } & \multirow{2}{*}{ 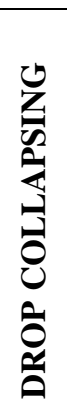 } & \multicolumn{5}{|c|}{$\begin{array}{l}\text { OIL DISPLACEMENT } \\
(\mathbf{m m})\end{array}$} & \multicolumn{5}{|c|}{$\begin{array}{l}\text { EMULSIFICATION } \\
\text { INDEX E24 (\%) }\end{array}$} & \multirow[t]{2}{*}{$\begin{array}{l}\text { NAME OF } \\
\text { BACTERIA }\end{array}$} \\
\hline & & & & $\begin{array}{l}\text { 预 } \\
\text { 至 } \\
0\end{array}$ & 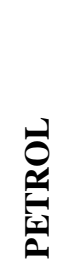 & 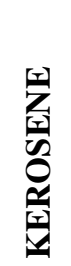 & 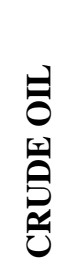 & 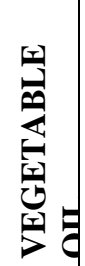 & $\begin{array}{l}\overrightarrow{|r|} \\
\text { 至 } \\
\overrightarrow{0}\end{array}$ & $\begin{array}{l}\overrightarrow{0} \\
\underline{y} \\
\underline{a}\end{array}$ & 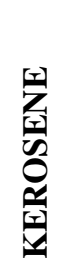 & 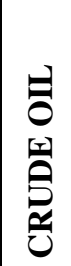 & 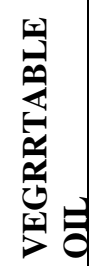 & \\
\hline N1 & + & - & + & 20 & 40 & 45 & 30 & 5 & 50 & 50 & 50 & 50 & 56 & $\begin{array}{l}\text { Staphylococcus } \\
\text { aureus }\end{array}$ \\
\hline $\mathrm{N} 2$ & + & - & + & 40 & 30 & \begin{tabular}{|l|}
17 \\
\end{tabular} & \begin{tabular}{|l|}
40 \\
\end{tabular} & 6 & 50 & 50 & 50 & 53 & 45 & Bacillus sp. \\
\hline N3 & - & - & + & 70 & 36 & 42 & 54 & 4 & 50 & 55 & 53 & 57 & 50 & $\begin{array}{l}\text { Pseudomonas } \\
\text { aeurignosa }\end{array}$ \\
\hline $\mathrm{N} 4$ & + & + & + & 15 & 30 & 66 & 52 & 5 & 61 & 50 & 53 & 54 & 58 & $\begin{array}{l}\text { Corynebacterium } \\
\text { sp. }\end{array}$ \\
\hline N5 & + & + & + & 32 & 30 & 62 & 60 & 5 & 56 & 47 & 50 & 50 & 55 & Escherichia coli \\
\hline N6 & + & - & - & 16 & 50 & 10 & 25 & 5 & 55 & 53 & 65 & 52 & 57 & Bacillus sp. \\
\hline N7 & + & - & + & 15 & 35 & 30 & 65 & 5 & 67 & 50 & 56 & 50 & 52 & $\begin{array}{l}\text { Pseudomonas } \\
\text { aeruginosa }\end{array}$ \\
\hline $\mathrm{N} 8$ & - & + & + & 85 & 45 & 12 & 42 & 4 & 50 & 53 & 56 & 53 & 58 & Bacillus sp. \\
\hline N9 & + & - & + & 25 & 34 & 7 & 69 & 4 & 60 & 40 & 59 & 52 & 57 & Bacillus sp. \\
\hline N10 & - & + & - & 75 & 30 & 20 & 65 & 8 & 62 & 50 & 53 & 50 & 73 & Streptococcus sp. \\
\hline N11 & + & - & + & 65 & 40 & 10 & 50 & 15 & 63 & 50 & 44 & 52 & 46 & Bacillus sp. \\
\hline N12 & - & + & + & 80 & 42 & 10 & 52 & 5 & 50 & 45 & 54 & 50 & 50 & Pseudomonas sp. \\
\hline N13 & + & - & + & 15 & 30 & 35 & 37 & 5 & 47 & 44 & 54 & 72 & 46 & Staphylococcus sp. \\
\hline N14 & - & - & - & 22 & 42 & 18 & 30 & 5 & 59 & 50 & 65 & 54 & 48 & Micrococcus sp. \\
\hline N15 & - & - & - & 15 & 52 & 22 & 42 & 5 & 55 & 47 & 70 & 57 & 52 & Staphylococcus sp. \\
\hline N16 & - & + & - & 52 & 18 & 20 & 40 & 5 & 61 & 47 & 64 & 50 & 52 & $\begin{array}{l}\text { Pseudomona } \\
\text { aeruginosa }\end{array}$ \\
\hline N17 & - & - & - & 60 & 32 & 18 & 60 & 4 & 65 & 47 & 58 & 52 & 59 & Pseudomonas sp. \\
\hline N18 & + & - & - & 30 & 28 & 14 & 30 & 4 & 65 & 45 & 61 & 56 & 48 & Pseudomonas sp. \\
\hline N19 & + & - & + & 60 & 38 & 16 & 48 & 5 & 59 & 56 & 58 & 40 & 52 & Bacillus sp. \\
\hline $\mathrm{N} 20$ & + & - & + & 30 & 36 & 16 & 67 & 3 & 54 & 43 & 56 & 67 & 54 & Bacillus sp. \\
\hline $\mathrm{N} 21$ & + & - & + & 30 & 35 & 12 & 57 & 10 & 61 & 41 & 61 & 37 & 45 & Enterobacter sp. \\
\hline $\mathrm{N} 22$ & + & - & + & 60 & 40 & 15 & 53 & 4 & 71 & 56 & 54 & 56 & 54 & Proteus sp. \\
\hline $\mathrm{N} 23$ & + & - & + & 13 & 25 & 10 & 61 & 17 & 61 & 53 & 58 & 72 & 56 & Pseudomonas sp. \\
\hline $\mathrm{N} 24$ & + & - & - & 75 & 50 & 10 & 62 & 4 & 55 & 50 & 54 & 50 & 52 & Proteus sp. \\
\hline
\end{tabular}

\section{Discussion:-}

In the study we collected crude oil sample, water sample from Otamiri river, swimming pool, fish pond, soil samples from palm oil mill, near kerosene pump, near diesel pump, near petrol pump, automobile soil sample, paint industry and milk sample. The total viable count from Table 1 shows very high viable counts with swimming pool sample the highest $\left(7.8 \times 10^{5} \mathrm{cfu}\right)$ followed by palm oil mill sample $\left(6.8 \times 10^{5} \mathrm{cfu}\right)$. The Tables 2,3,4 are the biochemical characteristics of the isolates. We observed different genera of micro organisms which are ubiquitous as shown in Table 1 and are not restricted to a particular environmental sample. The percentage abundance of bacteria genera isolated include Staphylococcus aureus 9.1\%, Bacillus spp. 21.2\%, Pseudomonas aeruginosa 27.3\%, Corynebacterium spp. 9.1\%, Streptococcus spp. 9.1\%, Micrococcus spp. 12.1\%, Enterobacter sp. 3.0\%, Proteus spp. 6.1\%, and Escherichia coli 3.0\%. Several researchers (Thenmozhi and Nagasathya, 2010; El-Sheshtawy., 2013; Tambekar and Gadakh, 2013; Okore et al., 2013) have isolated different Bacillus spp. and Pseudomonas spp. from crude oil and products contaminated soil and water samples. The result of this study is in agreement with the 
finding. The work of (Saisa-ard et al., 2013) reported the isolation of Bacillus spp. and Corynebacterium spp. from palm oil mill. This study also confirms their report. The other genera Staphylococcus aureus, Proteus spp., Streptococcus spp. Escherichia coli,Micrococcus spp. were isolated from contaminated water samples from swimming pool, fish pond, Otamiri river, abattoir and milk sample.

Based on the results on Table 5, we had the qualitative and the qualitative parts of the result. The qualitative result comprises the $\beta$ haemolysis, methylene blue agar assay and Drop collapsing method. There was a total of 16 isolates (66.7\%) that produced $\beta$ haemolysis (zone of clearance around the colony); 6 isolates (25\%) formed clear halos for methylene blue agar assay; 16 isolates $(66.7 \%)$ collapsed on a flat surface that was coated with oil in drop collapsing test out of the 24 isolates. The isolates that had positive results on two screening methods: haemolysis and drop collapsing are N1, N2, N7, N9, N11, N13, N19, N20, N21, N22, N23. The isolates that are positive for both drop collapsing and methylene blue agar assay are N8, N12. The isolates that had a positive result for the three qualitative tests i.e. the drop collapsing, haemolysis and methylene blue agar assay are the two isolates N4-Corynebacterium sp. and N5- Esherichia coli.

Blood agar lysis has been used to quantify lipopeptide/lipoprotein (surfactin) (Lin et al., 1998; Moran et al., 2002; Okore et al., 2013) and rhamnolipids (1qbal et al., 1995; Okore et al., 2013) and has been used to screen for biosurfactant production by new isolates. According to a research work by Youssef et al., (2004) $\beta$ haemlysis gave a large number of false positives and negatives. That report is in contrast to the findings in this current research as $66.7 \%$ of all isolates were positive for $\beta$ haemlysis test and their biosurfactant production ability was confirmed by drop collapse method that recorded also $66.7 \%$.

The ability of biosurfactant producers to form clear halos in methylene blue agar plate assay has also been recorded by several authorities (Lin et al., 1998; Govindammal and Parthasarathi, 2013; Maneerat, 2005; Siegmund and Wagner, 1991). That report is also confirmed by the finding in this report which recorded $25 \%$ positive for isolates for methylene blue agar plate assay.

Drop collapse method has been used to test for biosurfactant production by various authors (Jain et al., 1991; Bodour and Miller-Maier, 1998; Youssef et al., 2004; Maneerat and Phetrong, 2007; Satpute et al., 2008; Karthik et al., 2010; Thenmozhi and Nagasathya, 2010; Saravanan and Vijayakumar, 2012; Vanadana, 2012; Govindammal and Parthasarathi, 2013).

The quantitative part of the result comprises of the oil spreading and emulsification index method. The oil spreading test on diesel, the isolates that displayed highest diameter clear zone are $\mathrm{N} 8(85 \mathrm{~mm}$, Bacillus sp.) and N12 (80 mm, Pseudomonas sp.); on petrol N15 (52 mm, Staphylococcus sp.), N6 (50 mm, Bacillus sp.) and N24 (50 mm, Proteus sp.); on kerosene N4 (66 mm, Corynebacterium sp.), N5 (62 mm, Esherichia coli); on crude oil N9 (69 mm, Bacillus sp.), N10 (65 mm, Streptococcus sp.); on vegetable oil the zones of displacement recorded for all isolates were very low but $\mathrm{N} 23$ showed $17 \mathrm{~mm}$ (Pseudomonas sp.) while $\mathrm{N} 11$ gave $15 \mathrm{~mm}$ (Bacillus sp.). These genera (Proteus spp., Streptococcus spp., E.coli, Staphylococcus aureus) have scarcely been reported as biosurfactant producers. In this study they equally showed positive for screening tests, exhibited various zones of displacement on hydrocarbons used and high E24. Proteus sp. (75 mm oil displacement test, 71\% E24, +ve Bhaemolysis test, +ve oil collapse test), Streptococcus sp.(75 mm oil displacement, +ve methylene blue test), E.coli (62 mm oil displacement, $+\mathrm{ve}$ Bhaemolysis test, +ve oil collapse test, +ve drop collapse test), Staphylococcus $\mathrm{sp} .(72 \%$ E24, +ve $\beta$ haemolysis test, +ve drop collapse). Youssef et al., (2003) in their study on comparison of methods to detect biosurfactant production by diverse microorganisms found that, the oil spreading and drop collapse methods were correlated with the ability of the cultures to reduce surface tension. The oil spreading technique measures the diameter of clear zones caused when a drop of a biosurfactant containing solution is placed on an oil water surface. Morikawa et al., (2000) used this method to compare the activity of both cyclic and linear forms of surfactin and arthrofactin.

The highest emulsification index recorded by the isolates on the different hydrocarbons are: on diesel N22 (71 mm, Proteus sp), N7 (67 mm, Pseudomonas sp.);onpetrol, N19 and N22 (56 mm both, Bacillus sp. and Proteus sp.), N3(55 mm, Pseudomonas aeruginosa); on kerosene N15 (70 mm, Staphylococcus sp.), N14 and N6 (65 mm both, Micrococcus sp. and Bacillus sp.); on crude oil N23 and N13 (72 mm both, Pseudomonas sp. and Staphylococcus sp.), N20 (67 mm, Bacillus sp.); on vegetable oil N10 (73 mm, Streptococcus sp.), N8 (58 mm, Bacillus sp.). Proteus sp., Streptococcus sp., E.coli, Staphylococcus sp. equally emulsified the various hydrocarbons used. 
There is also a direct relationship between biosurfactant production and emulsifying activity according to Carillo $e t$ al., (1996); Fiebig et al., (1997); Youssef et al., (2004); Tabatabaee et al., (2005); Maneerat and Phetrong, (2007); Satpute et al., (2008); Anandaraj and Thivakaran, (2010); Karthik et al., (2010); Thenmozhi and Nagasathya, (2010); Saravanana and Vijayakumar, (2012); Vanadana, (2012); Govindammal and Parthasarathi, (2013). The result in Table 5 also confirms this report as the bacteria isolates that recorded high E24 values also showed high and positive values for the other screening tests for biosurfactant production.

\section{Conclusion and Recommendation:-}

In conclusion we isolated nine different genera of bacteria. They include Staphylococcus aureus $9.1 \%$, Bacillus sp. $21.2 \%$, Pseudomonas aeruginosa 27.3\%, Corynebacterium sp. 9.1\%, Streptococcus sp. 9.1\%, Micrococcus sp. $12.1 \%$, Enterobacter sp. 3.0\%, Proteus sp. 6.1\%, and Escherichia coli 3.0\%. The bacteria that showed high values for all screening tests were not restricted to a particular genus; virtually the genera isolated recorded high values for both E24 and oil displacement. These genera also gave positive results for $\beta$ haemolysis, methylene blue agar assay and Drop collapsing method. The diversity of these genera can be utilized in degrading different classes of hydrocarbons. Also an in-depth study on their genome and production quantities will make available such knowledge for mass production of these biosurfactant as alternative to chemical surfactants in remediation of environmental contaminants.

\section{Reference:-}

1. Anandaraj, B. and Thivakaran, P. (2010). Isolation and production of biosurfactant producing organisms from oil spilled soil. Biosci Tech. 1(3):120-126.

2. Bento, F.M., Fla'vio, A., De-Oliveira, C., Okeke, B.C. and Frankenberger Jr., W.T., (2005). Diversity of biosurfactant producing microorganisms isolated from soils contaminated with diesel oil. Microbiological Research. 160:249-255.

3. Bodour, A.A. and Miller-Maier, R.M. (1998). Application of a modified drop-collapse technique for surfactant quantitation and screening of biosurfactant producing microorganisms. J. Microbial Methods. 32:273-280.

4. Bredholt, H., Josefen, K., Vatland, A., Bruhbeim, P. and EImhjellen, K. (1998). Emulsification of crude oil by an alkane-oxidizing Rhodococcus species isolated from seawater. Can. J. Microbiol. 44: 330-340.

5. Desai, J.D., and Banat, I.M. (1997). Microbial production of surfactants and their commercial potential. Microbiological molecular Reviews. 61:47-64.

6. El-Sheshtawy, H.S., Aiad, I., Osman, M.E., Abo-ELnasr, A.A., and Kobisy, A.S. (2013). Comparative study of biosurfactants production by Bacillus licheniformis and Candida albicans for Microbial Enhanced Oil Recovery. Journal of Applied Sciences Research. 9(8):4877-4883.

7. Fiebig, Schulze, D., Chung, J.C., and Lee, S.T. (1997). Biodegradation of polychlorinated biphenys (PCBs) in the presence of a bioemulsifier produced on sunflower oil. Biodegration, 8:67-75.

8. Frielo, D.A., Mylroie, J.R., and Chakrabarty, A.M. (2001). Use of genetically engineered multi-plasmid microorganisms for rapid degradation of fuel hydrocarbons. Intern. Biodet. Biodeg. 48:233-242.

9. Govindammal, M., and Parthasarathi, R. (2013). Investigation on antimicrobial activity of biosurfactant produced by Pseudomonas fluorescens isolated from the mangrove ecosystem. International Research Journal of Pharmacy. 4(1):230-232 http://www.iriponline.com

10. Hassanshahian, M., Emtiazi, G., and Cappello, S. (2012). Isolation and characterization of crude oil degrading bacteria from the Perssian Gulf and Caspian sea. Mar Pollut bull. 64(1):7-12.

11. Igbal, S., Khalid, Z.M., and Malik, K.A. (1995). Enhanced biodegradation and emulsification of crude oil and hyper production of biosurfactants by a gamma ray induced mutant of Pseudomonas aeruginosa. Lett. Appl. Microbiol. 21:176-179.

12. Jain, D.K., Collins- Thomas, D.L., Lee, H., and Trevors, J.T. (1991). A drop collapsing test for screening surfactant producing microorganisms, J. Microbiol. Methods. 13:271-279.

13. Karthik, L., Kumar, G., and Bhaskara Rao, K.V., (2010). Comparison of methods and screening of biosurfactant producing marine Actinobacteria isolated from marine sediment. IIOAB Journal. 1(2):34-38.

14. Kiran, G.S., Thomas, T.A., Selvin, J., Sabarathnam, B., and Lipton, A.P. (2010). Optimization and characterization of a new lipopeptide biosurfactant produced by marine Brevibacterium aureum MSA13 in solid state culture. Bioresour. Technol. 101:2389-2396.

15. Lin, S.C., Lin, K.G., Lo, C.C., and Lin Y.M. (1998). Enhanced biosurfactant production by a Bacillus lichenformis mutant. Enzyme Microb. Technol. 23:267 - 273.

16. Maneerat, S. (2005). Biosurfactants from marine organisms. Songklanakarin. J.Sci Technolo. 27(6):1265-1272 
17. Maneerat, S. and Phetrong, K. (2007). Isolation of biosurfactant producing bacteria and characteristics of selected biosurfactant. Songklanakarin J. Sci. Technol. 29(3):781-791.

18. Minf, S., Chamkha, M., Labat, M., and Sayadi, S. (2011). Simultaneous hydrocarbon biodegradation and biosurfactant production by oil field-selected bacteria. J. Appl. Microbiol. 111:525-536.

19. Moran, A.C., Alejandra Martinez, M., and Sineriz, F. (2002). Quantification of surfactin in culture supernatant by haemolytic activity. Biotechnol. Lett. 24:177-180.

20. Morikawa, M., Hirata, Y., and Imanaka, T.A. (2000). Study on the structure, function and relationship of the lipopeptide biosurfactants. Biochem Biophys. Acta, 1488:211-218.

21. Okore, C.C., Mbanefo, O.N., Onyekwere, B.C., Onyewenjo, S., Abba-Father, C.A.M. (2013). Isolation and Characterization of Biosurfactants Producing Bacteria from Oil Polluted Soil",Journal of Natural Sciences Research www.iiste.org. ISSN 2224-3186 (Paper) ISSN 2225-0921 (Online). 3(5) pp 119-122

22. Pinto, M.P., Silva-Júnior, Jd.Se., Lima, A.Ad. and Grelle, C.E.V. (2014) multi-scales analysis of primate diversity and protected areas at a Megadiverse Region. PLoS ONE 9(8): e105205. ttps://doi.org/10.1371/journal.pone.0105205

23. Saisa-Ard, K., Maneerat, S., and Saimmai, A. (2013). Isolation and characterization of biosurfactantsproducing bacteria isolated from palm oil industry and evaluation for biosurfactants production using low-cost substrates. BioTechnologia. Journal of Biotechnology, Computational Biology and Bionanotechnology. 94(3):275-284.

24. Saisa-Ard, K., Maneerat, S. and Saimmai, A. (2013). Isolation and characterization of biosurfactantsproducing bacteria isolated from palm oil industry and evaluation for biosurfactants production using low-cost substrates. BioTechnologia. Journal of Biotechnology, Computational Biology and Bionanotechnology. 94(3):275-284.

25. Saravanan, V. and Vijayakumar, S. (2012). Isolation and screening of biosurfactant producing microorganisms from oil contaminated soil. J.Acad. Indus. Res. 1(5):264-268.

26. Satpute, S.K., Bhawasar, B.D., Dhakephalkar, P.K. and Chopade, B.A. (2008). Assessment of different screening methods for selecting biosorfactant producing marine bacteria. IndianJ. of marine science 37(3):243250.

27. Schulz, D., Passeri, A., Schmidt, M., Lang, S, Wagner, F., Wray, V. and Gunkel, W. (1991). Marine biosurfactants I. Screening for biosurfactants among crude oil degrading marine microorganisms from the North sea. Z. Naturefosch. 46c:197 - 203.

28. Siegmund, I. and Wagner, F. (1991). New method for detecting rhamnolipids excreted by Pseudomonas spp. during growth on mineral agar. Biotechnol. Tech. 5:265-268.

29. Tabatabaee, A., Mazaheri-Assadi, M., Nochi, A.A. and Sajadian, V.A. (2005). Isolation of biosurfactant producing bacteria from the oil reservoirs. Iranian J. Env. Health. Sci Eng. 2(1):6-12.

30. Tambekar, D.H. and Gadakh, P.V. (2013). Biochemical and molecular detection of biosurfactant producing bacteria from soil. Int. J. Life Sc. Biotechnology and Pharma. Research. 2(1):204-211.

31. Thenmozhi, R. and Nagasathya, A. (2010). Screening for surface active agent producing Bacteria in used in engine oil contaminated soil samples. Research J. of Agriculture and Biological sciences. 6(6):983-986.

32. Vandana, S. (2012). Biosurfactant-Isolation, Production, Purification and Significance. Intl. J. Scientific and Research Publications. 2(7):1-4.

33. Youssef, N.H., Duncan, K.E., Nagle, D.P., Savage, K.N., Knapp, R.M. and Mcinerney, M.T. (2004). Comparison of methods to detect biosurfactant production by diverse microorganism. Journal of Microbiological method, 56:339 - 349 .

34. Yuste, L., Corbella, M.E., Turiegano, M.J., Karison, U., Puyet, A. and Rojo, F. (2000). Characterization of bacteria strains able to grow on high molecular mass residues from crude oil processing. FEMS Microbial Ecol 32:69-75. 